\section{Ring Method for Measuring Surface Tension}

A GREAT deal has been written about the ring method since it was rescued from oblivion in 1919 . It is to-day more widely used than any other throughout the world. Let us try and analyse the reasons for its success.

The first two reasons are its rapidity and facility. Whoever has laboured over any of the other methods must admit this much. The third reason was the ruggedness of the instrument-a great asset in students' laboratories and lecture rooms. The fourth reason is the reliability of the method. It was primarily intended for biological fluids, and for the first time physiologists and biological chemists could check their measurements without trouble, rapidly. It was its remarkable reliability which induced such workers as Prof. Harkins to devote much of their valuable time to establish the correction formulæ, in collaboration with Dr. Jordan, and to compute the values of the factor $F$ for different dimensions of the ring, so as to make its readings more rigorous. A. Ferguson stated that of twenty odd methods only three were reliable: the ring method was one.

Its accuracy and reliability being established, its rapidity and ease of handling being well known, I still believe that the greatest reason for the success of the ring method was the fact that it proved to be constructive, inasmuch as it disclosed a certain number of new phenomena which were not altogether devoid of interest. I refer to the discovery that, in a solution, the establishment of equilibrium-the only conditions in which the Gibbs-Thomson thermodynamic equation is valid-is a slow process capable of being followed step by step; to the discovery of the factors governing the surface tension of protein and other solutions; of the recovery after lowering of the surface tension of such solutions by surface active substances, due to a mutual adsorption; of the existence of absolute minima ; of a method for determining the surface of adsorption of materials such as charcoal, graphite, platinum black, etc.; of a method for following the process of immunity; of a photo-capillary effect in plant sap ; of a method for detecting structural changes in serum proteins following inactivation; of a method for detecting alterations in transformer oil after use ; of a method for ascertaining the lubricating value of oils; and so on.

Now all these phenomena do not depend on absolute but on relative values. They were found because the method was very rapid, simple, reliable, and because it was possible to take into account the time factor. Relative methods are often more fruitful than absolute ones, as absolute values are of no particular interest except when dealing with pure substances. But I think everybody will agree, particularly the biologists, that solutions offer a broader field of investigation than pure liquids. As a matter of fact, the physical chemist and the physicist use comparatively few absolute methods.

Freud and Freud, however, succeeded in develop. ing a brilliant new theory which led them to accept the ring method as absolute, and this, in addition to the collection of new facts overlooked so far, and difficult, if not impossible, to study with another method, is another important reason why the ring method, in its modern form, has been so widely adopted in research as well as in industrial laboratories.

LECOMTE DU NoüY.

Institut Pasteur, Paris.

\section{Oxidation of Silane}

In view of a reference by Semenoff ${ }^{1}$ to some results recently obtained by Schantarowitsch on the upper and lower critical explosion limits in the oxidation of silane, it is of interest to record similar observations which we have made during the last six months.

We have found that pure silane when mixed with oxygen is not explosive at atmospheric pressure, but that when the pressure of the mixture is reduced, ignition occurs at a well-defined limit, which is higher the higher the temperature. The effect of two typical oxidation inhibitors (chloroform and ethyl iodide) has been examined. In small concentra. tions they lower the upper limit and in larger amounts prevent ignition altogether. This inhibiting action may be counteracted by increasing the temperature. Even a mixture containing the spontaneously inflammable higher hydrides of silicon may be rendered non-inflammable by small amounts of either of these inhibitors, and doubtless other substances will be found to have a similar action.

The lower oxidation limit of silane - oxygen mixtures has been examined in tubes of varying diameter, the walls of which were coated with a film of concentrated sulphuric acid. The following typical results in a cylindrical tube of $1.25 \mathrm{~cm}$. diameter show the order of magnitude of this lower pressure and the relation between the pressure of the separate gases at the limit :

$\begin{array}{lllllll}p_{\mathrm{SiH}_{4}} \text { (mm.) } & 0.837 & 0.605 & 0.368 & 0.272 & 0.241 & 0.198\end{array}$ $\begin{array}{llllllll}p_{\mathrm{O}_{2}} & \text { (mm.) } & 0.040 & 0.049 & 0.067 & 0.093 & 0.105 & 0.159\end{array}$

Under the conditions so far studied, the lower critical oxidation pressure shows a direct proportion. ality to a power of the vessel diameter slightly greater than unity, and not to the square of the diameter, as in the case worked out theoretically by Semenoff for deactivation on the vessel walls. On dry glass surfaces the lower limit is higher, but consistent results have not yet been obtained.

These results, which will be published in greater detail later, are in general accord with those of Schantarowitsch, as described by Semenoff, and show that the oxidation of silane resembles very closely that of phosphine rather than that of methane.

H. J. Emeléus. K. Stewart.

Imperial College of Science, London, S.W.7. Feb. 5.

1"Chemical Kinetics and Chain Reactions", 1935, p. 376.

\section{Reactivity of Carbon}

X-RAY examination ${ }^{1}$ indicates the presence of graphite in specimens of 'amorphous' carbon. $\mathrm{Y}$. Oshima and $Y$. Fukuda ${ }^{2}$ conclude that coke and charcoal consist of minute particles of graphite contaminated by and cemented together with hydrocarbon complexes. 'The so-called 'amorphous' forms of carbon generally show a chemical activity much greater than that displayed by graphite. It is, therefore, of interest to report a reaction in which the reverse is the case. During some systematic investigations of the reactivity of various types of cokes it was discovered that graded $(60-100$ I.M.M. mesh sieves), hard, metallurgical coke is oxidised at $100^{\circ}$ by excess of a mixture of chromic and 